\title{
OBSERVATION OF ALFVÉN EIGENMODES DRIVEN BY OFF-AXIS NEUTRAL BEAM INJECTION IN THE TCV TOKAMAK
}

\section{B. GEIGER ${ }^{1}$, A. N. KARPUSHOV ${ }^{2}$, P. LAUBER ${ }^{3}$, S. SHARAPOV ${ }^{4}$, M. DREVAL ${ }^{5}$, F.BAGNATO ${ }^{2}$, M. BAQUERO-RUIZ ${ }^{2}$, A. DAL MOLIN ${ }^{6}$, B.P. DUVAL ${ }^{2}$, M. GARCIA-MUNOZ 7, C. MARINI ${ }^{8}$, M. NOCENTE ${ }^{6}$, O. SAUTER ${ }^{2}$, L. STIPANI ${ }^{2}$, D. TESTA ${ }^{2}$, M. VALLAR ${ }^{2}$ AND THE TCV ${ }^{9}$ AND EUROFUSION MST1 ${ }^{10}$ TEAMS}

\author{
${ }^{1}$ UW Madison, Wisconsin, USA \\ ${ }^{2}$ Ecole Polytechnique Fédérale de Lausanne, Swiss Plasma Center, Lausanne, Switzerland \\ ${ }^{3}$ Max-Planck-Institut für Plasmaphysik, Boltzmannstr. 2, 85748 Garching, Germany \\ ${ }^{4}$ Culham Science Centre, Abingdon OX14 3EB, United Kingdom \\ ${ }^{5}$ Institute of Plasma Physics of the NSC KIPT, 61880 Kharkiv, Ukraine \\ ${ }^{6}$ Universita' di Milano-Bicocca, Piazza della Scienza 3, 20126, Milano, Italy \\ ${ }^{7}$ Univeristy of Seville, Spain \\ ${ }^{8}$ Oak Ridge Associated Universities, Tennessee, USA \\ 9 see the author list of "S. Coda et al, Nucl. Fusion 59, 112023 (2019)" \\ ${ }^{10}$ see the author list of "B. Labit et al, Nucl. Fusion 59, 086020 (2019)"
}

Email of corresponding author: benedikt.geiger@wisc.edu

\section{Abstract}

Fast-particle driven Alfvén Eigenmodes (AEs) have been observed in low-collisionality discharges with off-axis neutral beam injection (NBI), electron cyclotron resonance heating (ECRH) and a reduced toroidal magnetic field. During NBI and ECRH, Toroidicity induced Alfvén Eigenmodes (TAEs) appear in frequency bands close to $200 \mathrm{kHz}$, and chirping modes are observed at about 40 $\mathrm{kHz}$ and $80 \mathrm{kHz}$ that are likely Energetic-Particle-Induced Geodesic Acoustic Modes (EGAMs). When turning off ECRH in the experiment, those beam-driven modes disappear which can be explained by a modification of the fast-ion slowing down distribution. In contrast, coherent fluctuations close to the frequency of the beam driven TAEs are present throughout the experiment. The modes have the same toroidal mode number as the beam-driven ones and are even observed during ohmic plasma conditions. This clearly demonstrates that they are not caused by fast particles and suggests an alternative drive, such as turbulence. The mode-induced fast-ion transport has been found to be weak and marginal in terms of the fast-ion diagnostic sensitivities. Measurements of the plasma stored energy, neutron rates, neutral particle fluxes and fast-ion D-alpha spectroscopy show good agreement with neoclassical modelling result from TRANSP. This is further supported by a similarly good agreement between measurement and modelling in cases with and without 
ECRH and therefore with and without the modes. Instead, a significant level of charge exchange losses are predicted and observed which generate a bump-on-tail fast-ion distribution function that can provide free energy to EGAMs.

\section{Introduction}

Good fast-ion confinement is essential in fusion devices to ensure efficient alpha-particle heating and to protect the first wall from localized losses [1]. While neo-classical transport is typically negligible and below $0.1 \mathrm{~m}^{2} / \mathrm{s}$ [2], transport induced by MHD modes such as Alfvén Eigenmodes (AEs) has been observed under certain conditions to be well above $1 \mathrm{~m}^{2} / \mathrm{s}[3,4]$. Such strong fastion transport significantly impacts the fast-ion confinement and has to be avoided in future fusion devices. This in turn requires the development of reliable theoretical models that are validated in various regions of parameter space. At the "Tokamak à Configuration Variable" (TCV) [5, 6] the fast-ion transport can be investigated in a unique parameter space with very large fast-ion fractions. Thanks to the combination of a powerful $1 \mathrm{MW}(25 \mathrm{keV})$ neutral deuterium $\left(D^{0}\right)$ beam source [7] and a relatively small plasma volume of $1 \mathrm{~m}^{3}$ (TCV has minor and major radii of 0.25 and $0.88 \mathrm{~m}$, respectively), fast-ion densities can even exceed $50 \%$ of the electron density [8]. In addition, TCV is equipped with state-of-the-art fast-ion diagnostic, including FIDA spectroscopy [9], neutral particle analyzers [10] and neutron counters [11], and is therefore well suited to test fast-ion models and codes. This paper presents first experiments on neutral-beam driven AEs at TCV. The experimental setup and a representative plasma discharge are introduced in section two, and section three describes the observation of AEs based on magnetic and soft X-ray fluctuation measurements, as well as modelling results based on the linear gyrokinetic code LIGKA[12]. In section four, fast-ion measurements are compared with TRANSP/NUBEAM [13] modelling, and section five provides a short discussion and outlook.

\section{Experimental setup}

The experiments described here were performed with a plasma current of $\mathrm{I}_{\mathrm{p}}=120 \mathrm{kA}$ and a reduced toroidal magnetic field of $\mathrm{B}_{\mathrm{t}}=1.3 \mathrm{~T}$ (compared to $1.43 \mathrm{~T}$ ). A limiter configuration was chosen to maintain L-mode conditions throughout the experiment, and the plasma was shifted upwards $\left(z_{0}=+12 \mathrm{~cm}\right)$ for off-axis NBI. The neutral beam source injects tangentially in the $\mathrm{z}=0$ plane (see figure 1a), and upwards-shifting yields a fast-ion deposition profile that is peaked at a normalized toroidal flux label $\left(\rho_{t}\right)$ of 0.46 and, thus an off-axis peaked fast-ion distribution (see figure $1 \mathrm{~b}$ ).

Time traces of a representative experiment are plotted in figure 2. Starting at $0.7 \mathrm{~s}$, NBI heating is applied during four $120 \mathrm{~ms}$ long phases with $0.82 \mathrm{MW}$ of power delivered into the TCV vacuum vessel. The small spikes on top of the neutral beam heating power are due to a diagnostic neutral 

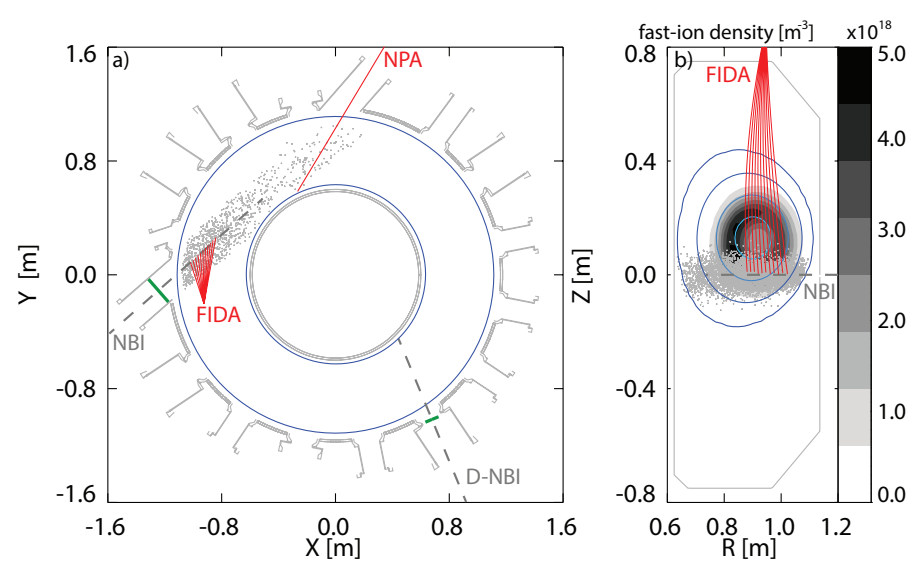

Figure 1: Top-down (a) and poloidal (b) views on the TCV tokamak. Deposition positions of NBI markers from TRANSP are drawn with gray points and the FIDA and NPA viewing geometries are illustrated in red. The gray contours in (b) show the fast-ion density during off-axis NBI and contours of the flux-surfaces are given in blue.

beam (DNB) [14] used for charge exchange recombination spectroscopy (CXRS). Between $0.5 \mathrm{~s}$ and $1.2 \mathrm{~s}$, the plasma was additionally heated by two $82.7 \mathrm{GHz}$ gyrotrons (X2) that deposit $0.4 \mathrm{MW}$ of ECRH power on the high-field-side at a normalized toroidal flux label $\left(\rho_{t}\right)$ of about 0.5. As displayed in figure $2 \mathrm{~b}$, the core-electron density was close to $4 \times 10^{19} / \mathrm{m}^{3}$ and increased to about $6 \times$ $10^{19} / \mathrm{m}^{3}$ when ECRH was turned off. The effective charge $\left(\mathrm{Z}_{\mathrm{eff}}\right)$ profile is considered flat at a value of 1.2 which increases to 1.5 during ECRH. This behavior has been inferred from comparisons between predicted and measured levels of the loop voltage (see section on TRANSP modelling) and is expected, as ECRH provides an enhanced level of physical sputtering from the carbon walls. The central electron temperature, as plotted in figure $2 \mathrm{~d}$, reaches up to $1 \mathrm{keV}$ and does not increase with the application of off-axis ECRH. In contrast, a clear increase of the electron temperature at $\rho_{t} \sim 0.5$ can be observed when ECRH is applied and slightly increased levels become apparent during NBI. Finally, the evolution of the ion temperature and rotation are depicted in figures $2 \mathrm{~d}+\mathrm{f}$ for $\rho_{t}=0.5$ and $\rho_{t}=0.9$. Here, it is interesting to note that the application of NBI has only a weak impact on the measured kinetic profiles, suggesting that the absorbed NBI power is low and or that the ion heat and momentum transport are strong.

Figure 3 shows comparisons between measured and fitted (modified hyperbolic tangent function [15]) kinetic profiles for two time-points in discharge \#62117. The experimental electron temperature and density profiles are measured by a vertically aligned Thomson scattering system which covers the whole plasma cross-section, even when shifting the plasma vertically up. In contrast, the impurity ion temperature and rotation is measured by CXRS using the diagnostic neutral beam (DNB). The latter is located in the $\mathrm{z}=0$ plane and thereby limits the radial coverage of ion-temperature and rotation measurements to $\rho_{t}>0.5$. A third order polynomial is used to extrap- 


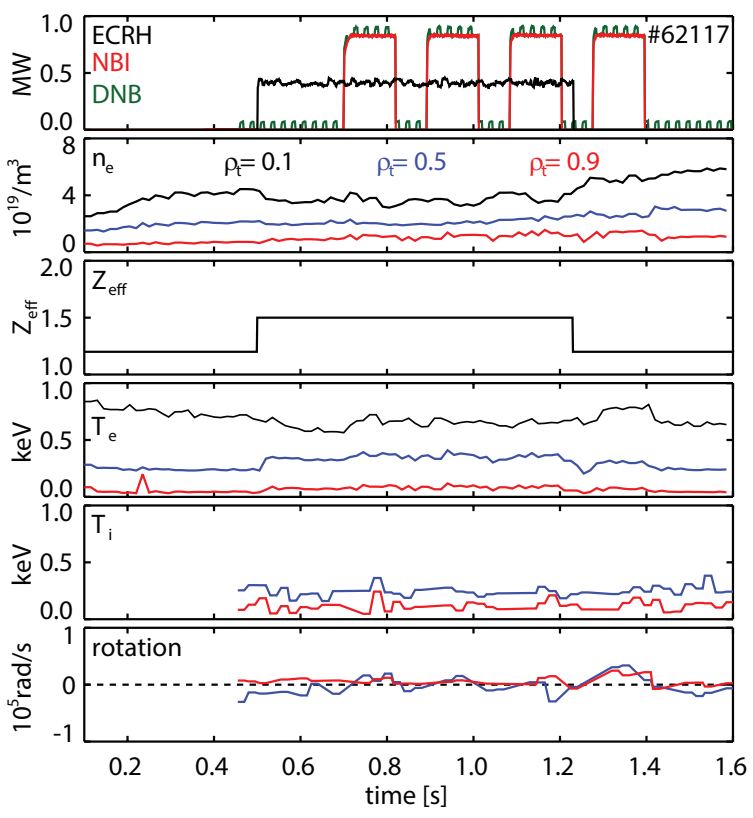

Figure 2: Representative time-traces of discharge \#62117.

olate the data towards the plasma core which provides large uncertainties that need to be considered when analyzing modelling results.

\section{Mode activity}

The experiments discussed here are rich in magneto-hydrodynamic mode (MHD) activity. This is in part explained by large fast-ion fractions (here, up to $20 \%$ of the electron density) and in part by the reduced magnetic field strength $(B=1.3 \mathrm{~T})$, which provides Alfvén velocities that are low enough for the excitation of TAEs through $25 \mathrm{keV}$ fast-ions. By considering an ion-density $n_{i}$ of $1.8 \times 10^{19} \mathrm{~m}^{-3}$ (this corresponds to the electron density at $\rho_{t}=0.5$, divided by $\mathrm{Z}_{\mathrm{eff}}=1.5$ ) and $\mathrm{B}_{t}=1.3 \mathrm{~T}$, the ratio of the fast-ion velocity, $v_{N B I}$, to the Alfvén velocity, $\mathrm{v}_{\mathrm{A}}$, reads:

$$
\frac{\mathrm{v}_{\mathrm{NBI}}}{\mathrm{v}_{\mathrm{A}}}=\frac{\sqrt{2 E / m_{D}}}{B / \sqrt{\mu_{0} m_{D} n_{i}}} \sim \frac{1.55 \times 10^{6} \mathrm{~m} / \mathrm{s}}{4.72 \times 10^{6} \mathrm{~m} / \mathrm{s}} \gtrsim \frac{1}{3}
$$

where $E$ is the fast-ion energy $(25 \mathrm{keV}), m_{D}$ is the deuterium mass and $\mu_{0}$ is the vacuum permeability. The resulting factor of $1 / 3$ is a well known threshold above which TAEs can be driven by circulating fast-ions through side-band excitation [16].

An overview of the mode activity using three different measurements is given in figure 4. Figures $4 a+b$ show poloidal and radial magnetic field fluctuations that are observed by high frequency LTCC-3D magnetic sensors [17], and figure 4c shows data from a core-channel of a soft X-ray 

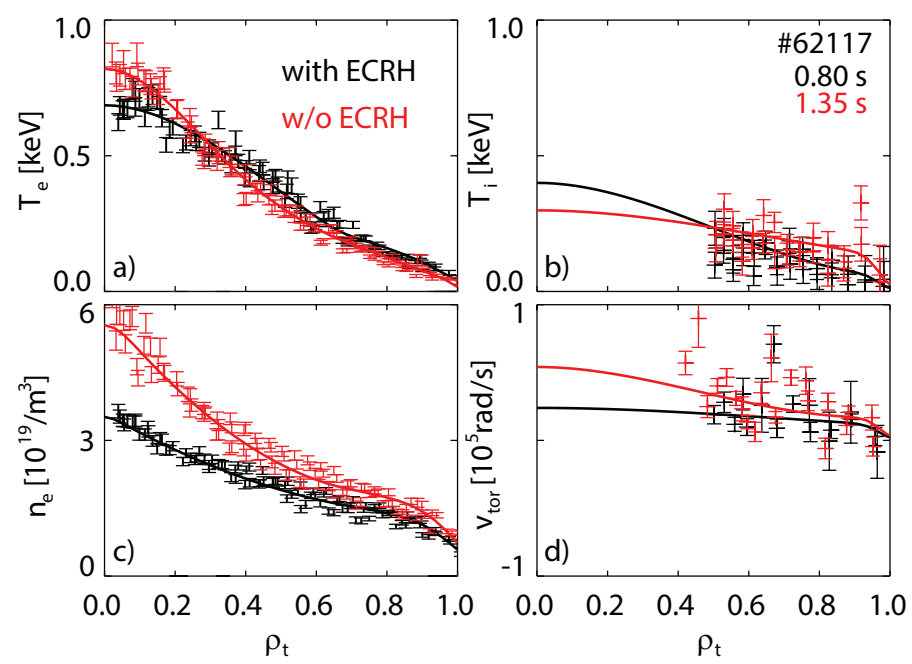

Figure 3: Kinetic profiles of \#62117 at $0.8 \mathrm{~s}$ and $1.35 \mathrm{~s}$.

camera system [18]. Enhanced mode-activity is clearly observed during the first three NBI phases with coherent fluctuations in the $200 \mathrm{kHz}$ and $40-100 \mathrm{kHz}$ regions.

\subsection{TAEs}

The modes present in the magnetic signals close to $200 \mathrm{kHz}$ have been identified as TAEs. The analysis of data from an array of toroidally distributed magnetic pickup-coils shows that the modes have a toroidal mode number of $n=3$. In addition, calculations of the expected TAE frequencies compare best with the experimental data for poloidal mode numbers $m=6$ and $m=5$. The expected TAE frequencies, as plotted in white in figure $4 a+b$, have been determined according to equations given in [19]:

$$
f_{T A E}[\mathrm{kHz}]=\frac{\mathrm{v}_{\mathrm{a}}}{4 \pi q R_{0}} \frac{\left|\left(2-\sqrt{1+\frac{3}{4} \epsilon_{g}^{2}}\right)\right|}{\sqrt{1-\frac{1}{4} \epsilon_{g}^{2}}} \cdot 10^{-3}
$$

with the major radius, $R_{0}$, and $\epsilon_{g}$ being related to the plasma elongation $\kappa$ via:

$$
\epsilon_{g}=\frac{\kappa^{2}-1}{\kappa^{2}+1}
$$

Finally, $q$ is the local safety factor which is defined for TAE modes as [20]:

$$
q=(m+0.5) / n
$$

According to equation 4, the q-value of a given TAE is defined by it's toroidal and poloidal mode numbers. Based on this q-value, the radial mode position (as a function of $\rho_{t}$ ) is then determined 


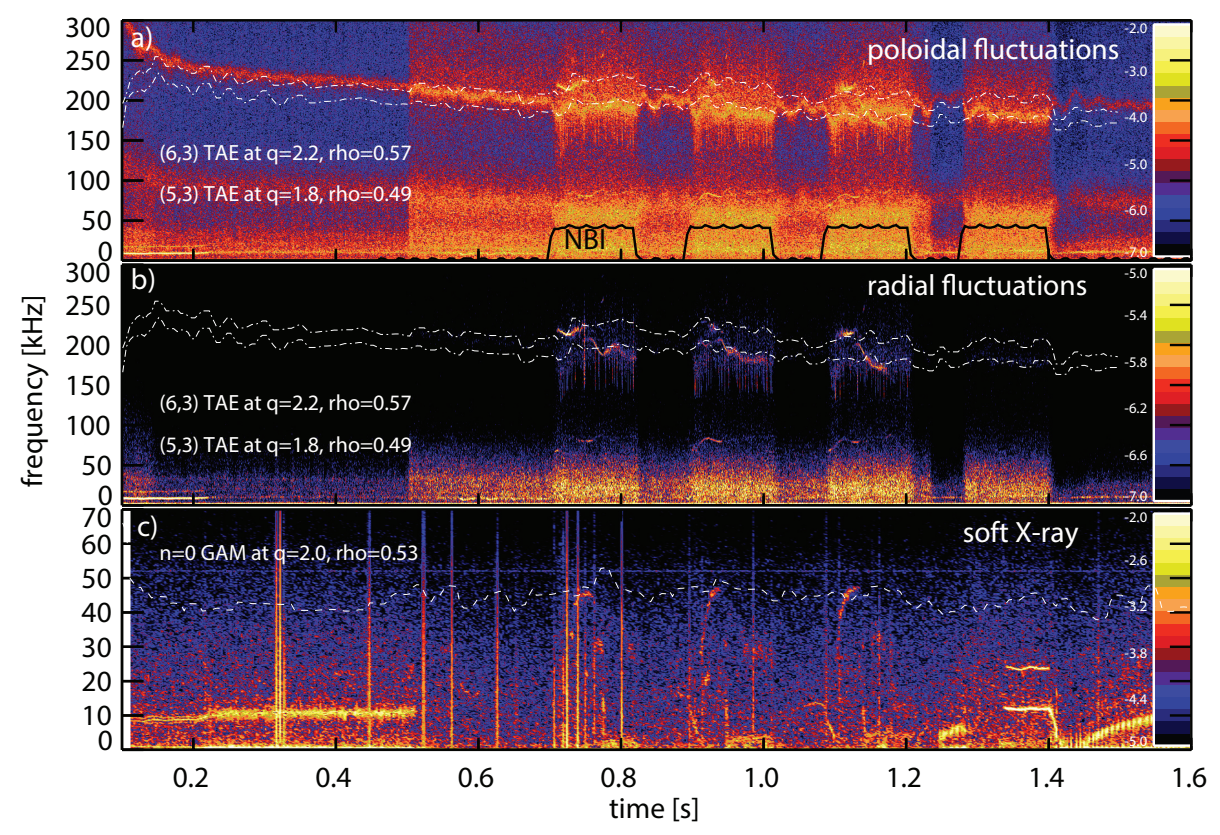

Figure 4: Spectrogram of poloidal (a) and radial (b) flucatations measured by magnetic pick-up coils, compared with the expected frequencies for $n=3, m=5$ and $n=3, m=6$ TAEs. c) Spectrogram of data from a soft X-ray channel, compared with the expected frequency of a GAM at the $\mathrm{q}=2$ surface.

using equilibrium information provided by TRANSP. Thus, the corresponding temperature, density and $\kappa$ values can be obtained, as required to calculate $\mathrm{v}_{\mathrm{A}}$ and the mode frequency $f_{T A E}$.

The calculated frequencies for $m=5$ and $m=6$ modes $(n=3)$ agree very well with the observed frequencies. In particular during NBI, those modes are observed in the poloidal and radial fluctuation data. This observation also agrees with simulations from the linear gyrokinetic code LIGKA[12]. LIGKA has been supplied with kinetic profiles and the TRANSP-predicted equilibrium as inputs and calculates the Alfvén continuum [21] (blue crosses in figure 5), as well as the global TAE mode structure of the least damped mode in the TAE gap for the experimentally given toroidal mode number. The resulting mode-amplitude profiles for $n=3$ TAEs are given in figure 5 in red for different poloidal mode numbers. As can be seen, the strongest mode amplitudes appear at $\rho_{t} \sim 0.5$ where a band-gap is present in the Alfvén continuum in order to minimise continuum damping. Interestingly, coherent fluctuations at the expected TAE frequencies can be seen in the poloidal fluctuation data well before the application of NBI heating. The modes are already present during the ohmic plasma (until $0.5 \mathrm{~s}$ ), become more intense during pure ECRH, and get even stronger when NBI is additionally switched on. This shows that the modes are driven more unstable by NBI but cannot be purely beam-driven ones. A similar observation has been made during ohmic plasmas at ASDEX Upgrade where the presence of TAEs could be attributed to plasma turbulence [20]. This 


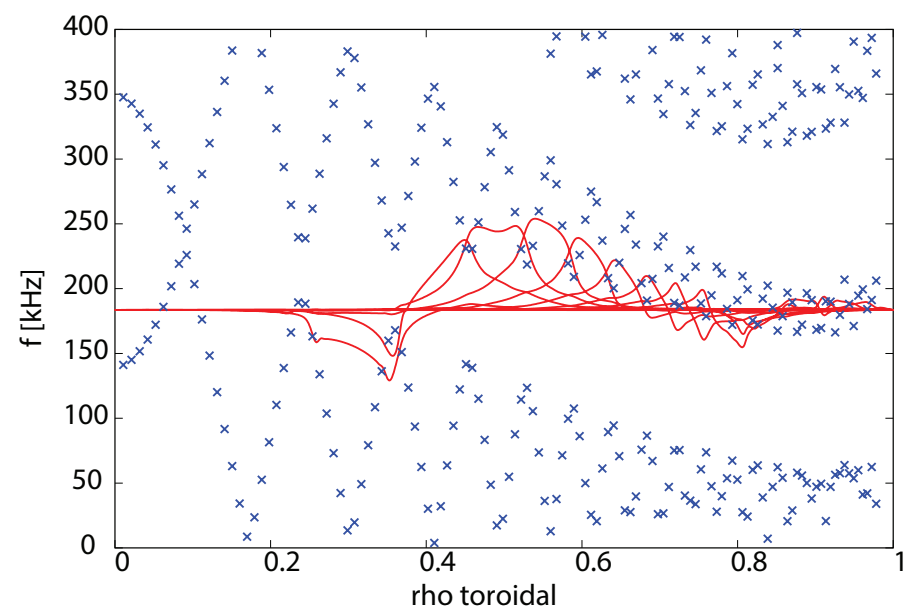

Figure 5: Predicted Alfvén continuum (blue crosses) and radial mode structure of $n=3$ TAEs from LIGKA as a function of $\rho_{t}$.

situation might be similar at TCV where plasmas are typically affected by turbulent fluctuations that develop from unstable trapped electron modes (TEM) and electron temperature gradient (ETG) modes [22]. In addition, it should be noted that such an interaction between turbulence and TAEs has recently been observed and studied in non-linear gyrokinetic GENE simulations that suggest a reduction of the turbulence level via coupling to TAEs [23]. However, it should be noted that in contrast to the local GENE simulations, the observed mode numbers are low, e.g. the destabilised TAEs seem to be global.

The observations from the poloidal and radial magnetic sensors are quite different. While the poloidal sensors pick-up the supposedly turbulence induced modes, the radial sensor only observes the beam-driven ones. This suggests different polarization directions of the modes and will serve as an important input for comparisons with theoretical and numerical results.

\subsection{EGAMs}

Upwards chirping modes at about $40 \mathrm{kHz}$ are observed during NBI by the soft X-ray system (called Duplex Multiwire Proportional soft X-ray counter, DMPX). Figure 4c shows data from one representative channel where modes can be identified shortly after turning on NBI. The data is compared with the expected frequencies of an $n=0$ Geodesic Acoustic Mode (GAM), located at the $q=2$ surface. The GAM frequency has been calculated using [19]:

$$
f_{\mathrm{GAM}}[\mathrm{kHz}]=\frac{\mathrm{v}_{\mathrm{A}}}{2 \pi R}\left[\left(2+\frac{1}{q^{2}}\right) \frac{2}{\kappa^{2}+1} \beta_{s}\right]^{2} \cdot 10^{-3}
$$


with

$$
\beta_{s}=\frac{c_{s}^{2}}{v_{A}^{2}}
$$

where $c_{s}$ is the sound velocity. The good agreement between the predicted and observed frequency and the chirping character of the modes strongly suggests that these are energetic particle driven GAMs (EGAMs) [24, 25].

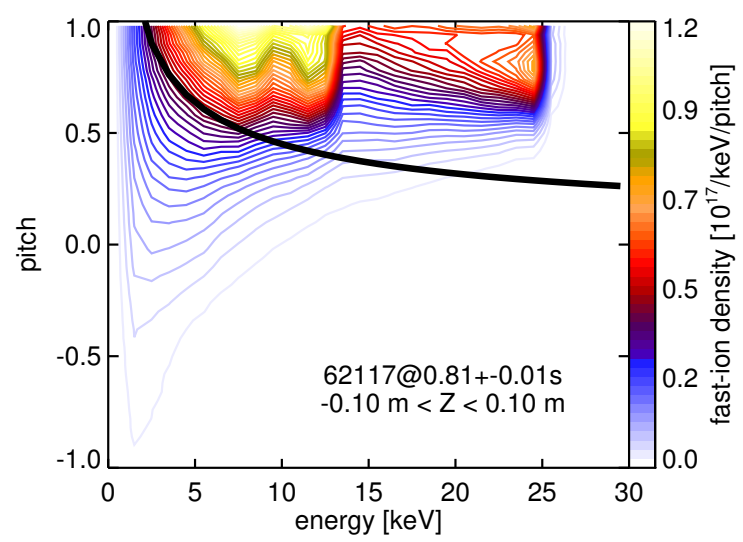

Figure 6: Fast-ion distribution as a function of energy and pitch compared to the pitch-energy resonance line at which $40 \mathrm{kHz}$ EGAMs might be driven. .

In addition, EGAMs are expected to be driven by bump-on tail fast-ion distribution functions which are present in TCV due to strong charge-exchange losses (discussed later). Figure 6 displays a TRANSP-predicted fast-ion distribution function for \#62117 as a function of energy, $E$, and pitch $=v_{\|} / v_{t o t}$ with $v_{\|}$being the velocity parallel to the magnetic field lines and the total velocity $v_{t o t}$. The fast-ion distribution function consists of mainly passing fast ions, peaks at the three injectionenergies $(25 \mathrm{keV}, 12.5 \mathrm{keV}$ and $8.3 \mathrm{keV})$ and features positive gradients in E. This provides free energy to excite the $n=0$ EGAMs that cannot be driven through radial gradients. In addition, figure 6 shows the location of possible wave-particle interactions in black which are given by:

$$
p(E)=\frac{f_{\mathrm{EGAM}} 2 \pi R q}{v_{\mathrm{NBI}}(E)}
$$

By employing the observed frequency $f_{\mathrm{EGAM}}=40 \mathrm{kHz}$, a resonance line $(p(E))$ is obtained that overlaps significantly with the fast-ion distribution function for $E<15 \mathrm{keV}$ where strong velocity space gradients are present.

Finally is should be noted that the $n=0$ character of EGAMs cannot be addressed by the magnetic sensors since these appear to be insensitive to the fluctuations. This might be in part explained by broadband-noise in the magnetic data below $50 \mathrm{kHz}$ and in part by rather localized radial Eigenfunctions of those modes. In contrast, there are $n=0$ modes observed in the LTCC-3D data at about 
$80 \mathrm{kHz}$ (see figure 4). For these modes, however, it is not clear whether they are EGAMs, as the expected frequencies (equation 5) would only match for core-localized modes (near q=1) where strong damping is expected. Unfortunately, the modes are not seen in data from the soft X-ray system due to bandwidth-problems which restricts our analysis to about $70 \mathrm{kHz}$.

\subsection{Absence of Alfvén modes without ECRH}

During the 4th NBI phase in discharge \#62117 (1.28 s-1.4 s), ECRH was turned off. Interestingly this phase does not exhibit NBI-driven fluctuations, which might be explained by either increased damping (stability) or by a reduced drive of the modes. In particular for the TAEs, the damping seems not to be affected significantly since the coherent fluctuations from the possibly turbulencedriven modes are still present (see figure $4 a$ ).
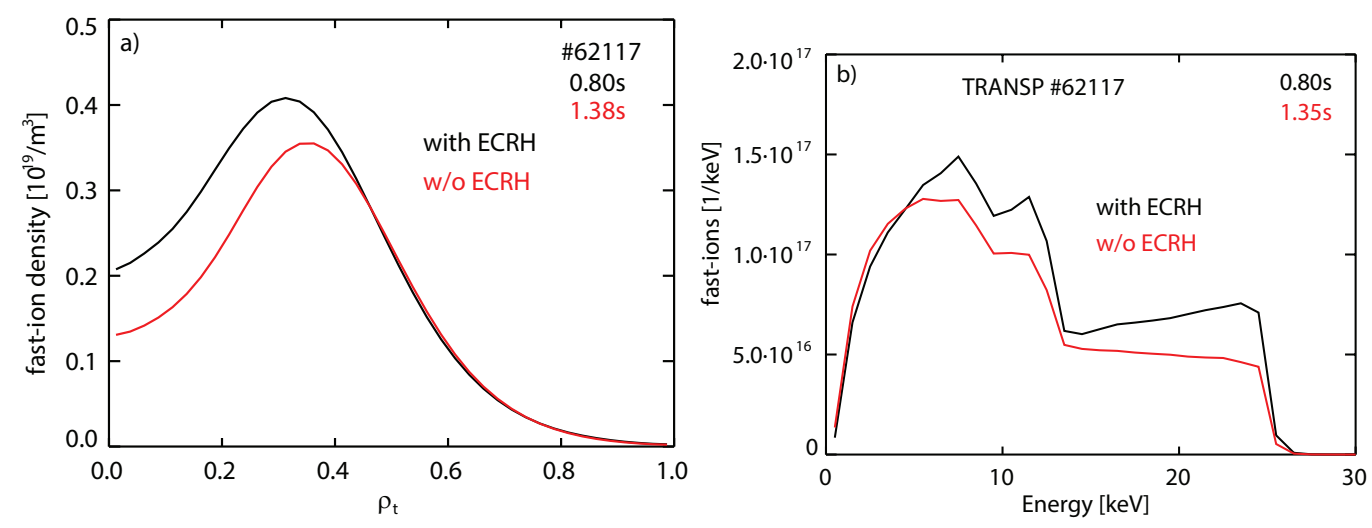

Figure 7: TRANSP predicted radial fast-ion density profile with (black) and without (red) ECRH.

b) TRANSP predicted energy distribution with (black) and without (red) ECRH.

In contrast, the slowing down time is reduced when switching off ECRH as the electron temperature decreases and the electron density peaking increases. This reduces the off-axis fast-ion density and the bump-on tail character. Figure 7 compares TRANSP-predicted fast-ion distribution functions as a function of $\rho_{t}$ and energy for the cases with and without ECRH. The predicted fast-ion density is clearly lower without ECRH and features a reduced gradient at $E$. A similar effect has e.g. been reported by [26] where ICRF-driven TAEs were observed only when applying additional ECRH, which enhanced the slowing down time.

\section{TRANSP modelling}

The fast-ion densities shown above have been calculated using the TRANSP code. The code has

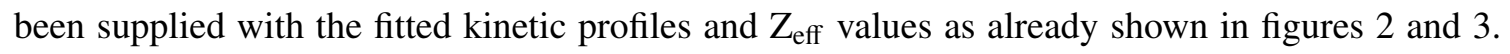
In addition, information on the NBI and ECRH systems, the evolution of the plasma boundary, the 
central magnetic field strength, the plasma current and the background neutral density have been provided.

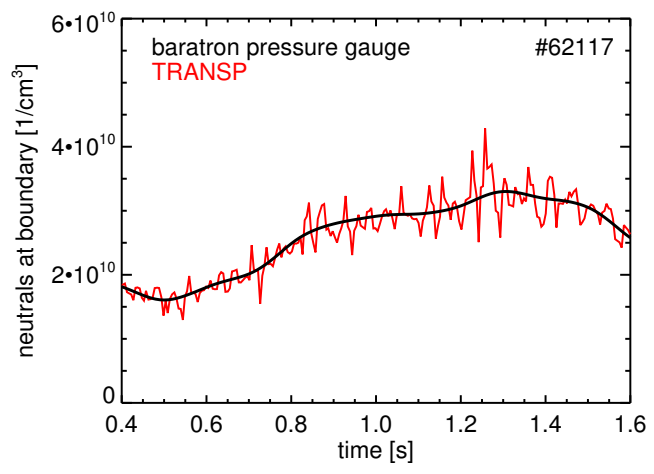

Figure 8: Comparison between the predicted edge neutral density from TRANSP/FRANTIC and the one inferred from a pressure gauge (baratron).

Careful treatment of the neutral density is important for TRANSP simulations of TCV plasmas since charge exchange losses alter the fast-ion density significantly [8]. Here, the FRANTIC module of TRANSP has been used to determine the shape of a 1D neutral density profile used in TRANSP. The absolute level of this profile is set by defining a global particle confinement (see also [8]), which has been chosen such that the resulting edge-neutral density matches experimental data deduced from a baratron pressure gauge (see figure 8).

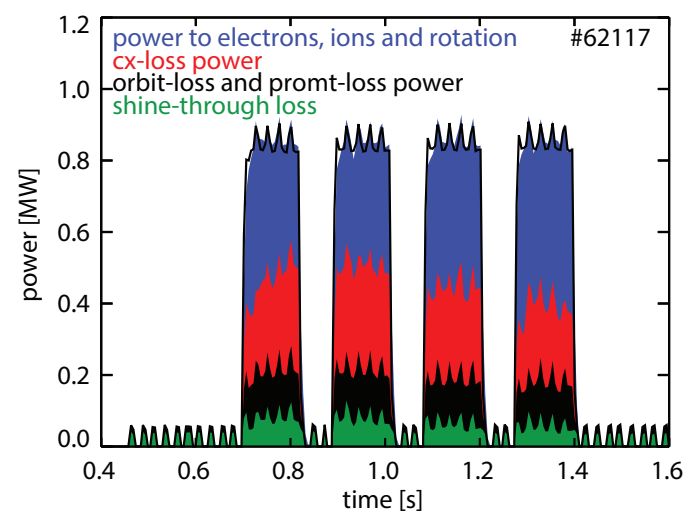

Figure 9: TRANSP-predicted distribution of the injected NBI power into heating and rotation input (blue), charge-exchange losses (red), orbit losses (black) and shine-through (green).

The predicted edge-neutral density is about $3 \times 10^{16} / \mathrm{m}^{3}$ and causes, in combination with the offaxis NBI population, strong charge-exchange losses. Figure 9 illustrates the distribution of the injected NBI power into plasma heating and momentum input (blue), as well as losses which sum 
up to about $50 \%$ of the input power. The losses consist of charge exchange losses (red), orbit losses (black) and shine-through (green). The contribution of charge exchange is particularly strong for off-axis injection in TCV since the small minor radius of TCV allows the neutral density to reach half radius without strong attenuation (only about a factor of 10). Under these conditions, the characteristic time for charge exchange is smaller than the slowing-down time such that the beam distribution function does not relax to the slowing-down form. In consequence a fast-ion energy distribution with positive gradients is obtained (see figures 6 and $7 b$ ).

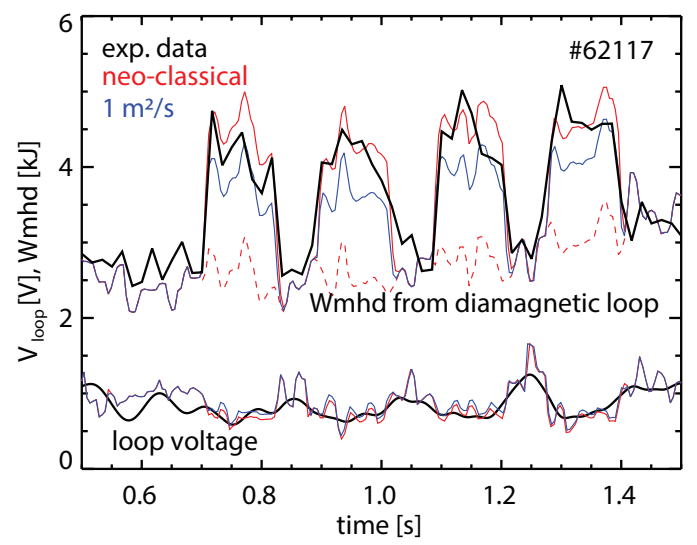

Figure 10: Measured loop voltage (around $1 \mathrm{eV}$ ) and plasma stored energy (about $5 \mathrm{~kJ}$ ) compared with predictions from TRANSP. The simulation in red corresponds to the assumption of neo-classical fast-ion transport while the simulation in blue refers to a global anomalous transport of $1 \mathrm{~m}^{2} / \mathrm{s}$. The dashed red line shows the simulated evolution of the plasma stored energy without considering fast particles.

Figure 10 shows a comparison of TRANSP predictions with measurements of the loop voltage and of the plasma stored energy ( $3 / 2$ of the perpendicular energy as measured by a diamagnetic loop). As can be seen, modelling and experiment are in good agreement which shows that the kinetic profiles and the corresponding calculation of the fast-ion pressure and current drive are consistent. During each NBI phase, the plasma stored energy increases. This increase is due to the additional presence of fast particles while the stored energy from the thermal plasma only (dashed line) is expected to remain constant. Here, it should be noted that the uncertainties of the central iontemperature are large. However, the plasma stored energy is weighted towards the outer radii since the core part contributes only with a small fraction of the plasma volume. Concerning the loop voltage, we adjusted the $Z_{\text {eff }}$ value in TRANSP to match the level of the loop voltage. Thus, it is no surprise that we obtain an overall good agreement between measurement and modelling. However, the simulation even follows the NBI-induced variations of the loop voltage, which suggest that the fast-ion current drive is modelled consistently. In addition to the neo-classical simulation in red, figure 10 shows (in blue) results from a simulation considering an ad-hoc fast-ion diffusion of 
$1 \mathrm{~m}^{2} / \mathrm{s}$. While the assumption of additional anomalous transport hardly changes the predicted loopvoltage, the predicted plasma stored energy is lower and slightly under-estimates the measurement.

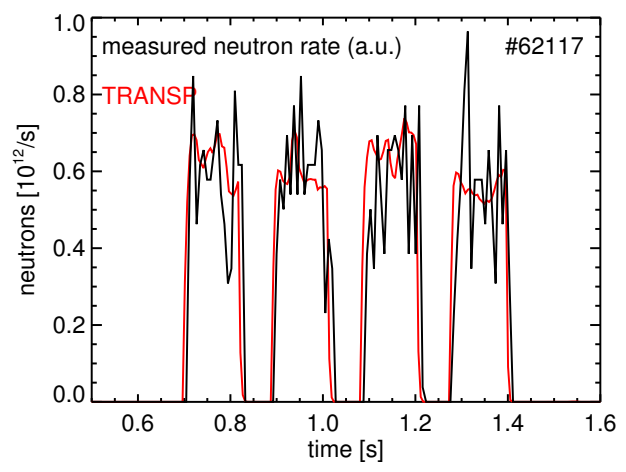

Figure 11: Measured neutron rates compared to a neo-classical TRANSP simulation (red).

In addition, the evolution of the fast ion density from TRANSP can be validated by the comparison with neutron rates, radial FIDA intensity profiles and NPA measurements. Experimental neutron rates are plotted in figure 11 . The experimental data is not absolutely calibrated and has been scaled to the neo-classical TRANSP simulation (red) by one factor such that only the evolution of the simulation and measurement can be evaluated. As can be seen, measurement and simulation agree well during all four phases which allows us to conclude that a possible anomalous transport induced by the beam-driven modes (not considered by TRANSP) is below the measurement sensitivity. If there was a significant loss of fast-ion confinement during the first three NBI phases, we would see enhanced neutron rates during the fourth phase when the modes are gone (without ECRH).

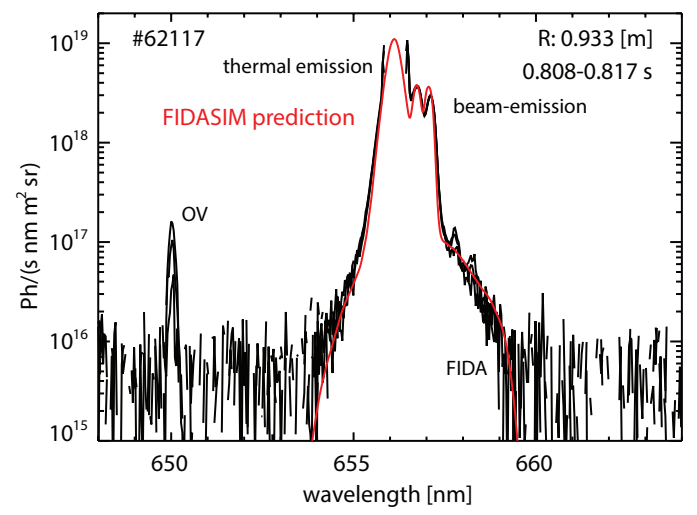

Figure 12: Exemplary FIDA spectrum of \#62117 compared with a synthetic spectrum from FIDASIM (red) which corresponds to the neo-classical TRANSP simulation.

A similar observation is made when analyzing data from fast-ion D-alpha (FIDA) spectroscopy. 


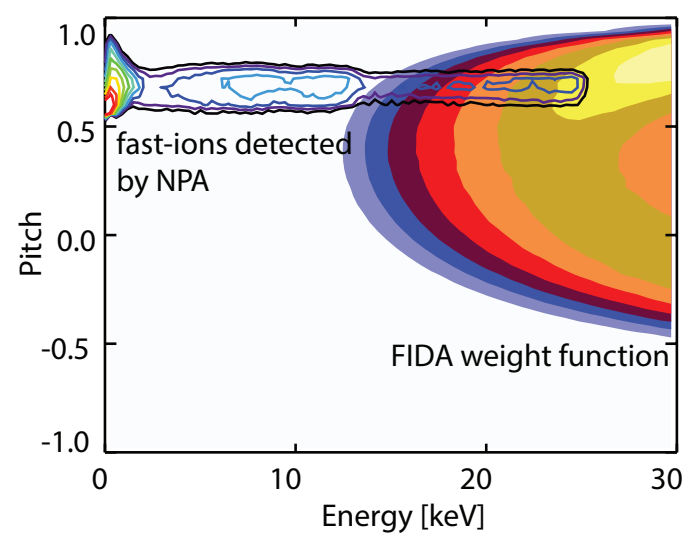

Figure 13: FIDA weight function (filled contour plot) and energy-pitch distribution of fast-ions being detected by the CNPA diagnostic (solid contours).

The FIDA system at TCV uses an array of lines of sight that intersect the neutral beam from topdown at different radial positions (see figure 1). Four representative spectra of a central channel are plotted in figure 12. Passive radiation, measured after turning off NBI, has been subtracted such that the spectra mainly consist of active contribution: the beam-emission, the thermal charge-exchange emission (halo) and the contribution from neutralized fast ions (FIDA light). Due to the NBI and observation geometries, mainly red-shifted FIDA radiation can be analyzed. The part of velocity space covered by studying FIDA radiation between $658.5 \mathrm{~nm}$ and $659.5 \mathrm{~nm}$ is depicted in figure 13 using a weight function [27]. The weight-function shows that the diagnostic provides information on fast ions with energies above $12 \mathrm{keV}$ and dominantly positive pitch values.

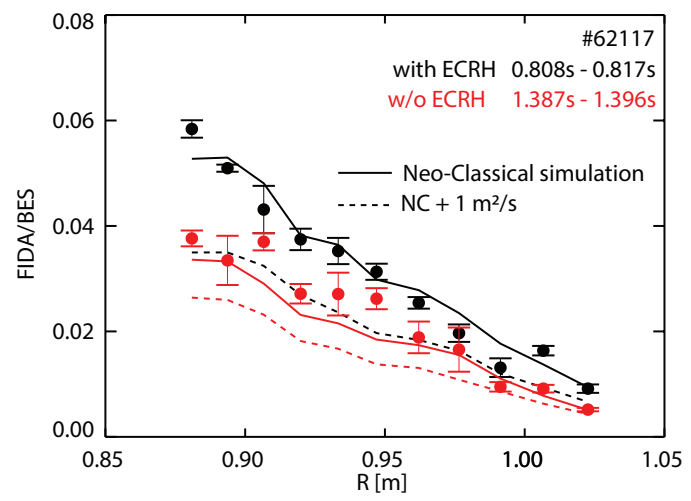

Figure 14: Radial FIDA intensity profiles with (black) and without ECRH (red). Corresponding neo-classical TRANSP/FIDASIM simulations are plotted with solid lines while simulations assuming anomalous transport are shown with dashed lines.

Radial FIDA profiles are obtained by integrating the FIDA emission of each line of sight in the 
specified wavelength interval $(658.5 \mathrm{~nm}-659.5 \mathrm{~nm})$. By further dividing the FIDA emissivity by the observed level of the beam emission, FIDA/BES profiles are obtained which are independent of an intensity calibration and permit comparison with modelling results from FIDASIM [28]. FIDASIM uses TRANSP predicted fast-ion distribution functions as input and provides synthetic spectra of the beam, halo and FIDA emission that can be integrated to obtain synthetic profiles. Figure 14 shows a comparison of predicted and measured radial FIDA/BES profiles for a time-point with ECRH ( $\sim 0.8 \mathrm{~s})$ and a time-point without ECRH $(\sim 1.38 \mathrm{~s})$. As can be seen, the measurements and neo-classical simulations (solid lines) agree very well . In addition, the FIDA/BES profile is higher during the phase with ECRH (red) which is expected due to modified kinetic profiles. The dashed lines in figure 14 represent synthetic profiles resulting from the TRANSP simulation assuming additional anomalous fast-ion transport of $1 \mathrm{~m}^{2} / \mathrm{s}$. These profiles clearly under-estimate the measurement and demonstrate that possible anomalous transport is well below this level.

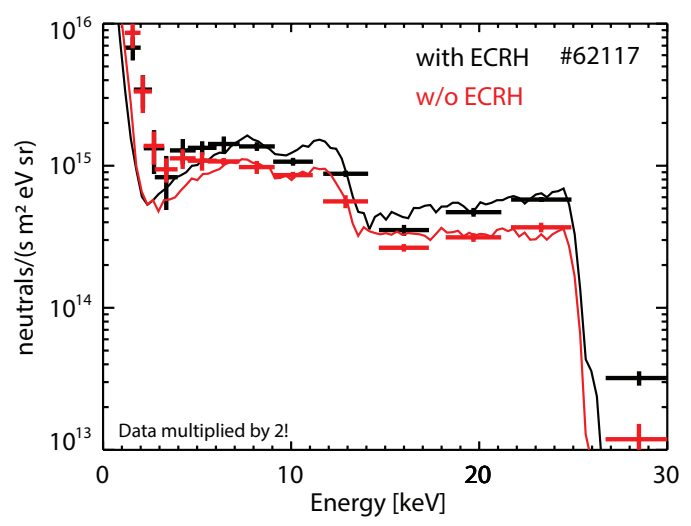

Figure 15: Energy spectra from the NPA detector compared with FIDASIM modelling results.

In addition, FIDASIM has been used to obtain synthetic neutral particle analyzer (NPA) measurements that can be compared with the measurements of the compact NPA system of TCV. The viewing geometry of the NPA is illustrated in figure 1 and the part of velocity space accessed by the NPA is depicted in figure 13. Energy spectra measured by the NPA detector for time points with and without ECRH are shown in figure 15. During ECRH, the fast-ion part of the signal $(\mathrm{E}>2 \mathrm{keV})$ is higher and slightly more peaked towards the injection energy than what is measured without ECRH. This behaviour is reproduced by FIDASIM, suggesting that the predicted fast-ion distribution function from TRANSP is valid. However, it should be noted that the data has been scaled by a factor of two in order to match the simulation. This factor might be explained by a degradation of the NPA detector or by a neutral density that is much smaller along the NPA view than what is expected by TRANSP/FRANTIC. Nevertheless, the shape and trend measured by the NPA diagnostic are well reproduced by the modelling. During ECRH, the measurement is considerably higher and features a stronger gradient towards higher energies than what is seen without 
ECRH. This again supports the conclusion that the mode-induced fast-ion transport is relatively weak.

\section{Summary and outlook}

The fast-ion confinement has been investigated in off-axis NBI experiments at TCV with a reduced magnetic field strength of $1.3 \mathrm{~T}$. For the first time, clear NBI-induced Alfvén eigenmode activity has been observed which consists of TAEs and EGAMs. While the EGAMs are driven by a bumpon tail fast-ion distribution function present due to strong charge exchange losses, the TAEs occur in a gap-structure around mid-radius and are even partially present during ohmic and pure ECRH plasmas. This suggests that the TAEs can be excited by additional mechanisms, such as turbulence. In addition, the NBI-driven modes are only present when off-axis ECRH is on, as demonstrated by one NBI phase without additional electron heating. This observation is likely explained by reduced fast-ion densities due to shorter slowing down times. The fast-ion measurements agree very well with this hypothesis and demonstrate that the fast-ion confinement is close to its neoclassical level. Moreover, the observed modes do not alter the fast-ion confinement to a noticeable level as no confinement-improvement is seen during the phase without TAEs and EGAMs. This experimental demonstration could already be a test case for codes that predict anomalous fast-ion transport and shows that, in presence of only few modes, the fast-ion transport remains weak. However, this situation might change with the planned installation of a second $50 \mathrm{kV}$ neutral beam which produces fast ions with velocities closer to the Alfven velocity. Moreover, the installation of divertor baffles [29] will reduce the neutral content in the plasma such that the fast-ion fraction can be further increased, again providing stronger Alfvén Eigenmodes to be studied.

\section{ACKNOWLEDGMENTS}

We would like to thank Prof. William Heidbrink for his help in interpreting the observed mode activity during his stay in Lausanne. This work has been carried out within the framework of the EUROfusion Consortium and has received funding from the Euratom research and training programme 2014 - 2018 and 2019 - 2020 under grant agreement No 633053. The views and opinions expressed herein do not necessarily reflect those of the European Commission.

\section{References}

[1] A. Fasoli, C. Gormenzano, H. Berk, B. Breizman, S. Briguglio, D. Darrow, N. Gorelenkov, W. Heidbrink, A. Jaun, S. Konovalov, R. Nazikian, J.-M. Noterdaeme, S. Sharapov, K. Shinohara, D. Testa, K. Tobita, Y. Todo, G. Vlad, and F. Zonca, "Chapter 5: Physics of energetic ions," Nuclear Fusion, vol. 47, pp. S264-S284, jun 2007.

[2] W. Heidbrink and G. Sadler, "The behaviour of fast ions in tokamak experiments," Nuclear Fusion, vol. 34, pp. 535-615, apr 1994. 
[3] M. Turnyanskiy, C. Challis, R. Akers, M. Cecconello, D. Keeling, A. Kirk, R. Lake, S. Pinches, S. Sangaroon, and I. Wodniak, "Measurement and control of the fast ion redistribution on MAST," Nuclear Fusion, vol. 53, p. 053016, apr 2013.

[4] C. S. Collins, W. W. Heidbrink, M. E. Austin, G. J. Kramer, D. C. Pace, C. C. Petty, L. Stagner, M. A. Van Zeeland, R. B. White, and Y. B. Zhu, "Observation of critical-gradient behavior in alfvéneigenmode-induced fast-ion transport," Phys. Rev. Lett., vol. 116, p. 095001, Feb 2016.

[5] A. Fasoli, "Overview of physics research on the TCV tokamak," Nuclear Fusion, vol. 49, p. 104005, sep 2009.

[6] A. Fasoli, "TCV heating and in-vessel upgrades for addressing DEMO physics issues," Nuclear Fusion, vol. 55, p. 043006, mar 2015.

[7] A. N. Karpushov, S. Alberti, R. Chavan, V. I. Davydenko, B. P. Duval, A. A. Ivanov, D. Fasel, A. Fasoli, A. I. Gorbovsky, T. Goodman, V. V. Kolmogorov, Y. Martin, O. Sauter, A. V. Sorokin, and M. Toussaint, "Upgrade of the tcv tokamak, first phase: Neutral beam heating system," Fusion Engineering and Design, vol. 96-97, pp. 493 - 497, 2015. Proceedings of the 28th Symposium On Fusion Technology (SOFT-28).

[8] B. Geiger, A. N. Karpushov, B. P. Duval, C. Marini, O. Sauter, Y. Andrebe, D. Testa, M. Marascheck, M. Salewski, P. A. Schneider, and and, "Fast-ion transport in low density 1-mode plasmas at TCV using FIDA spectroscopy and the TRANSP code," Plasma Physics and Controlled Fusion, vol. 59, p. 115002 , sep 2017.

[9] W. W. Heidbrink, K. H. Burrell, Y. Luo, N. A. Pablant, and E. Ruskov, "Hydrogenic fast-ion diagnostic using Balmer-alpha light," Plasma Physics and Controlled Fusion, vol. 46, pp. 1855-1875, nov 2004.

[10] A. N. Karpushov, B. P. Duval, C. Schlatter, V. I. Afanasyev, and F. V. Chernyshev, "Neutral particle analyzer diagnostics on the tcv tokamak," Review of Scientific Instruments, vol. 77, no. 3, p. 033504, 2006.

[11] X. Xufei, M. Nocente, F. Bonomo, P. Franzen, M. Frschle, G. Grosso, F. Grnauer, R. Pasqualotto, M. Tardocchi, T. Fan, and G. Gorini, "Neutron measurements from beam-target reactions at the elise neutral beam test facility," Review of Scientific Instruments, vol. 85, no. 11, p. 11D864, 2014.

[12] P. Lauber, S. Guenter, A. Koenies, and S. Pinches, "Ligka: A linear gyrokinetic code for the description of background kinetic and fast particle effects on the mhd stability in tokamaks," Journal of Computational Physics, vol. 226, no. 1, pp. 447 - 465, 2007.

[13] A. Pankin, D. McCune, R. Andre, G. Bateman, and A. Kritz, "The tokamak monte carlo fast ion module nubeam in the national transport code collaboration library," Computer Physics Communications, vol. 159, no. 3, pp. 157 - 184, 2004.

[14] A. N. Karpushov, G. Abdrashitov, I. Averboukh, P. Bosshard, I. Condrea, B. Duval, A. Ivanov, V. Kolmogorov, J. Mlynar, A. Perez, I. Shikhovtsev, A. Shukaev, and H. Weisen, "Upgrade of the diagnostic neutral beam injector for the tcv tokamak," Fusion Engineering and Design, vol. 66-68, pp. 899 - 904, 2003. 22nd Symposium on Fusion Technology. 
[15] R. J. Groebner and T. H. Osborne, "Scaling studies of the high mode pedestal," Physics of Plasmas, vol. 5, no. 5, pp. 1800-1806, 1998.

[16] H. Biglari, F. Zonca, and L. Chen, "On resonant destabilization of toroidal alfvn eigenmodes by circulating and trapped energetic ions/alpha particles in tokamaks," Physics of Fluids B: Plasma Physics, vol. 4, no. 8, pp. 2385-2388, 1992.

[17] D. Testa, A. Corne, G. Farine, C. Jacq, T. Maeder, and M. Toussaint, "3d, ltcc-type, high-frequency magnetic sensors for the tcv tokamak," Fusion Engineering and Design, vol. 96-97, pp. 989 - 992, 2015. Proceedings of the 28th Symposium On Fusion Technology (SOFT-28).

[18] A. Sushkov, V. Andreev, Y. Camenen, A. Pochelon, I. Klimanov, A. Scarabosio, and H. Weisen, "Highresolution multiwire proportional soft x-ray diagnostic measurements on tcv," Review of Scientific Instruments, vol. 79, no. 2, p. 023506, 2008.

[19] O. P. Fesenyuk, Y. I. Kolesnichenko, and Y. V. Yakovenko, "Frequencies of the geodesic acoustic mode and alfvn gap modes in high-q2 plasmas with non-circular cross section," Physics of Plasmas, vol. 20, no. 12, p. 122503, 2013.

[20] M. Maraschek, S. Günter, T. Kass, B. Scott, H. Zohm, and A. U. Team, "Observation of toroidicityinduced alfvén eigenmodes in ohmically heated plasmas by drift wave excitation," Phys. Rev. Lett., vol. 79, pp. 4186-4189, Nov 1997.

[21] P. Lauber, M. Brüdgam, D. Curran, V. Igochine, K. Sassenberg, S. Günter, M. Maraschek, M. GarciaMunoz, N. Hicks, and the ASDEX Upgrade Team, "Kinetic Alfvén eigenmodes at ASDEX Upgrade," Plasma Physics and Controlled Fusion, vol. 51, no. 12, p. 124009, 2009.

[22] G. Merlo, M. Fontana, S. Coda, D. Hatch, S. Janhunen, L. Porte, and F. Jenko, "Turbulent transport in tcv plasmas with positive and negative triangularity," Physics of Plasmas, vol. 26, no. 10, p. 102302, 2019.

[23] A. D. Siena, T. Grler, E. Poli, A. B. Navarro, A. Biancalani, and F. Jenko, "Electromagnetic turbulence suppression by energetic particle driven modes," Nuclear Fusion, vol. 59, p. 124001, sep 2019.

[24] G. Y. Fu, "Energetic-particle-induced geodesic acoustic mode," Phys. Rev. Lett., vol. 101, p. 185002, Oct 2008.

[25] L. Horváth, G. Papp, P. Lauber, G. Por, A. Gude, V. Igochine, B. Geiger, M. Maraschek, L. Guimarais, V. Nikolaeva, and G. P. and, "Experimental investigation of the radial structure of energetic particle driven modes," Nuclear Fusion, vol. 56, p. 112003, jul 2016.

[26] S. E. Sharapov, M. Garcia-Munoz, M. A. V. Zeeland, B. Bobkov, I. G. J. Classen, J. Ferreira, A. Figueiredo, M. Fitzgerald, J. Galdon-Quiroga, D. Gallart, B. Geiger, J. Gonzalez-Martin, T. Johnson, P. Lauber, M. Mantsinen, F. Nabais, V. Nikolaeva, M. Rodriguez-Ramos, L. Sanchis-Sanchez, P. A. Schneider, A. Snicker, and P. V. and, "The effects of electron cyclotron heating and current drive on toroidal alfvén eigenmodes in tokamak plasmas," Plasma Physics and Controlled Fusion, vol. 60, p. 014026, nov 2017.

[27] M. Salewski, B. Geiger, D. Moseev, W. W. Heidbrink, A. S. Jacobsen, S. B. Korsholm, F. Leipold, J. Madsen, S. K. Nielsen, J. Rasmussen, M. Stejner, and M. Weiland, "On velocity-space sensitivity of FIDA spectroscopy,” Plasma Physics and Controlled Fusion, vol. 56, p. 105005, 2014. 
[28] W. W. Heidbrink, D. Liu, Y. Luo, E. Ruskov, and B. Geiger, "A code that simulates fast-ion d-alpha and neutral particle measurements," Communications in Computational Physics, vol. 10, no. 3, p. 716741, 2011.

[29] H. Reimerdes, S. Alberti, P. Blanchard, P. Bruzzone, R. Chavan, S. Coda, B. Duval, A. Fasoli, B. Labit, B. Lipschultz, T. Lunt, Y. Martin, J.-M. Moret, U. Sheikh, B. Sudki, D. Testa, C. Theiler, M. Toussaint, D. Uglietti, N. Vianello, and M. Wischmeier, "Tcv divertor upgrade for alternative magnetic configurations," Nuclear Materials and Energy, vol. 12, pp. 1106 - 1111, 2017. Proceedings of the 22nd International Conference on Plasma Surface Interactions 2016, 22nd PSI. 